\title{
High frame-rate fluorescence confocal angle-resolved linear dichroism microscopy
}

\author{
Xiao Wang, ${ }^{1,2,3}$ Alla Kress, ${ }^{1,2,3}$ Sophie Brasselet, ${ }^{1,2,3}$ and Patrick Ferrand ${ }^{1,2,3, a)}$ \\ ${ }^{1}$ Aix-Marseille Université, Institut Fresnel, F-13013 Marseille, France \\ ${ }^{2}$ CNRS, Institut Fresnel, UMR 7249, F-13013 Marseille, France \\ ${ }^{3}$ Centrale Marseille, Institut Fresnel, F-13013 Marseille, France
}

(Received 30 January 2013; accepted 5 May 2013; published online 28 May 2013)

\begin{abstract}
Angle-resolved linear dichroism is a recent technique that exploits images recorded using an illumination field whose polarization angle is sequentially rotated during acquisition. It allows to retrieve orientation information of the fluorescent molecules, namely the average orientation angle and the amplitude of the fluctuations around this average. In order to boost up the acquisition speed without sacrificing the axial sectioning, we propose to combine a spinning disk confocal excitation scheme together with an electrooptical polarization switching and a camera acquisition. The polarization distortions induced when passing through the spinning disk system have been quantified and effectively compensated. The signal to noise features of the camera have been analyzed in detail so that the precision of the method can be quantified. The technique has been successfully tested on giant unilamellar vesicles and on living cells labeled with different fluorescent lipid probes, $\mathrm{DiIC}_{18}$ and di-8-ANEPPQ. It was able to acquire precise orientation images at full frame rates in the range of a second, ultimately limited by the fluorophore brightness and the camera sensitivity. @ 2013 AIP Publishing LLC . [http://dx.doi.org/10.1063/1.4807318]
\end{abstract}

\section{INTRODUCTION}

Polarization is a property of light that is widely exploited in life science microscopy for studying the structural organization of proteins and lipids using fluorescence probes. This relies on the fact that (i) the absorption efficiency of a fluorescent molecule is sensitive to the relative orientation between its absorbing dipole and the exciting electric field, and (ii) the emitted fluorescence light is polarized according to the direction of its emitting dipole.

The large variety of techniques based on these properties can be summarized into two main classes. First, so-called fluorescence anisotropy methods use a given polarization for excitation and are based on the analysis of the polarized emitted fluorescence. Because they are sensitive to depolarization process such as energy transfer, rotational diffusion, they have been first introduced to investigate molecules in solutions. ${ }^{1}$ Later, they have been used to address the issue of the organization of biomolecular assemblies., ${ }^{2,3}$

A second class of techniques exploits the change of absorption efficiency for different polarization conditions, namely the linear dichroism (LD). Because they are known to be weakly affected by depolarization processes, ${ }^{4,5}$ they are widely used to probe orientation of molecules, in order to address a vast variety of issues, including cholesterol depletion in membranes, ${ }^{4}$ orientational distributions of lipid probes, ${ }^{6}$ and conformal changes of proteins. ${ }^{7}$ In practice, LD usually involves a comparison of the fluorescence response recorded for two orthogonal directions of polarization of excitation. Measurements can be carried out using onephoton $^{5}$ or multiphoton ${ }^{4,6,7}$ absorption processes, using single point ${ }^{5-7}$ or multiplexed ${ }^{4}$ laser scanning. The data analysis can

\footnotetext{
a)Electronic mail: patrick.ferrand@ fresnel.fr
}

be performed either by post-processing two images that have been acquired sequentially, ${ }^{4}$ or by a real-time hardware ${ }^{5}$ or software $^{7}$ demodulation of a signal recorded using a fast polarization two-state switching at each scanning point.

While fluorescence anisotropy and LD have helped to solve important questions related to the structural organization of proteins and lipids, both techniques are based on two orthogonal directions of measurements and suffer from limitations because the observation volume usually addresses more than one emitter. In this case indeed, the description of the system cannot be given by a single orientation value and needs to introduce a geometrical model and a more complete set of parameters, for instance, the average orientation of the fluorophores and the width of the orientation distribution of the ensemble, an information that cannot be retrieved exhaustively by two measurements only. The usual approach has been to simplify the problem by restricting it to areas of samples where the orientation is appropriate, ${ }^{2,3,7,8}$ or to study specifically samples of perfectly controlled geometry, ${ }^{6,9,10}$ so that various orientations can be addressed in a controlled fashion. Another possibility would be to repeat the same measurement on a rotated sample, ${ }^{5}$ which is not convenient in practice. For these reasons, it considerably reduces the range of explorations and fails to grasp the whole complexity of molecular organization in heterogeneous samples.

Recently, a more refined mode of operation of LD measurement has been proposed, in order to circumvent these limitations. In this scheme that we summarize here under the generic term of angle-resolved LD, the sample is probed using several excitation polarization angles denoted $\alpha$, as illustrated in Fig. 1(a), and an unpolarized detection. DeMay et al. have reported ${ }^{11}$ on the use of four sequentially acquired fluorescence images, which allowed to quantify rapidly ( $0.3 \mathrm{fps}$ ) both orientation and anisotropy of septin 
(a)

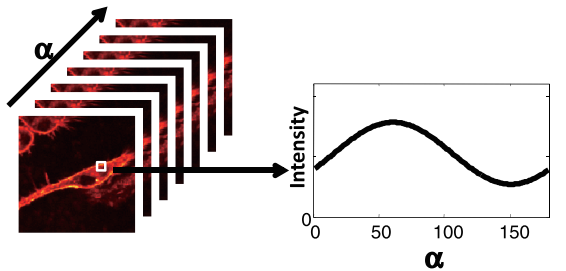

(b)

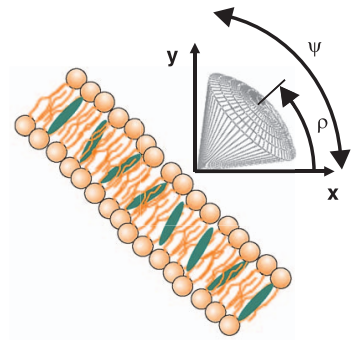

(c)

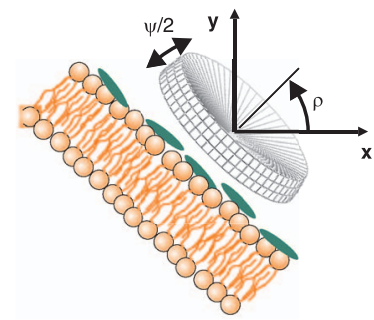

(d)

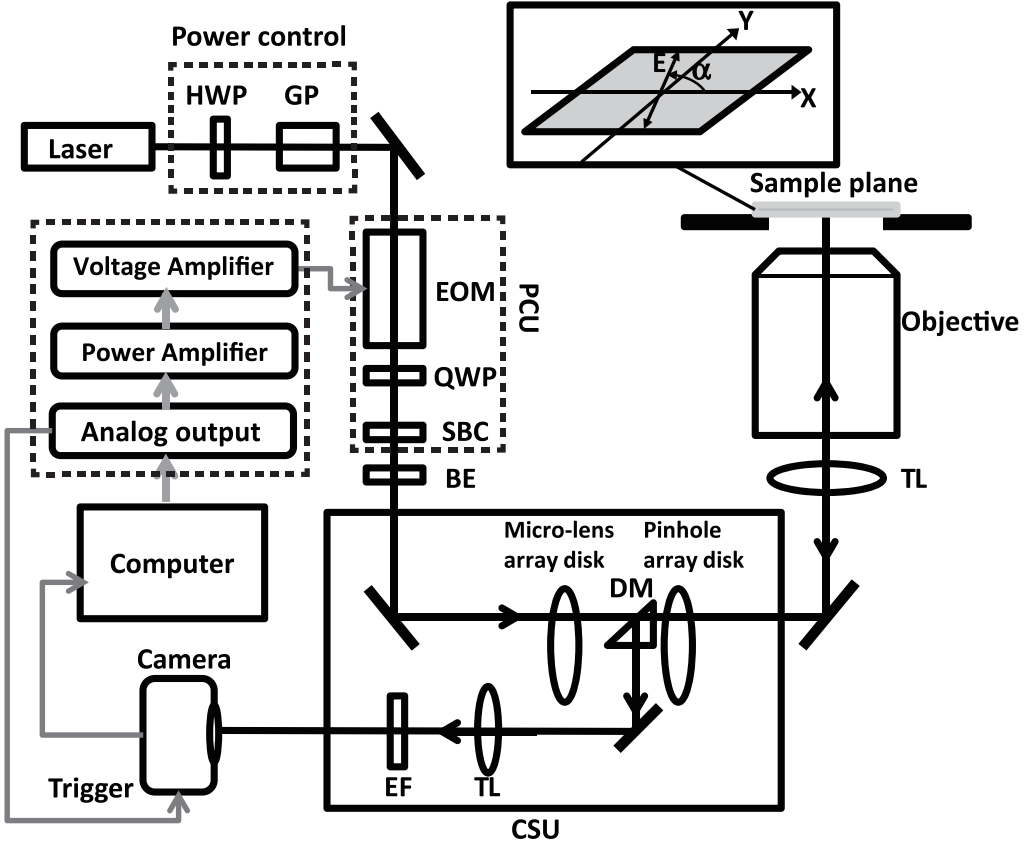

FIG. 1. (a) Principle of angle-resolved LD acquisition. An image stack is recorded for various polarization angles $\alpha$. The plot is an example of dataset extracted at one pixel. (b) The orientational distribution of fluorescent probes that insert perpendicularly to the membrane can be modeled as a $3 \mathrm{D}$ cone, defined by two angles $\rho$ and $\psi$. (c) The case of probes that lie on the membrane surface can be modeled as a pancake-like distribution, defined by two angles $\rho$ and $\psi$, too. (d) Experimental setup. The inset shows the linear polarization state of the exciting light, making an angle $\alpha$ in the sample plane. HWP: half wave plate, GP: Glan polarizer, EOM: electrooptic modulator, QWP: quarter wave plate, SBC: Soleil-Babinet compensator, BE: beam expander, DM: dichroic mirror, TL: tube lens, EF: emission filter, PCU: polarization control unit; CSU: confocal spinning disk unit.

proteins in live yeast, without any a priori orientational knowledge, a clear improvement as compared to conventional LD. However, in order to avoid polarization distortions induced by a dichroic mirror, the reported work used a wide field trans-illumination configuration, which restricts the technique to thin samples, causes photo-bleaching, and does not provide axial sectioning. An epi-fluorescence scheme, still based on wide field geometry, has been proposed by Lesoine et $a l .{ }^{12}$ However, experimental data recorded on controlled samples do not show the expected results yet and still require, according to the authors, further investigations. Based on the same principle as the above-mentioned methods, we have proposed a scheme by adapting a laser scanning confocal epi-fluorescence microscope ${ }^{13}$ in which the polarization distortion induced by the dichroic mirror and scanning galvanometers has been fully controlled. Using a cone (see Fig. 1(b)) or a pancake-like model (see Fig. 1(c)) as comprehensive descriptions for the distribution of orientation of the fluorophore, we were able to map the local organization of cell membranes ${ }^{13}$ and to monitor the polymorphism of amyloid fibrils. ${ }^{14}$ However, the measurement scheme that was chosen for these proofs of principle was based on point-bypoint scanning for image acquisition and mechanical control of the input polarization, so that it was highly time-consuming and, therefore, limited to static samples.

In this article, we propose a dramatic improvement of the acquisition speed of angle-resolved LD using a highly parallel confocal image system and fast polarization switching, without sacrificing the axial sectioning provided by confo- cal microscopy. For this purpose, we propose to perform the image acquisition using a high-speed spinning disk confocal unit. This system is capable of generating and scanning patterns of thousands excitation spots in the specimen, that are analyzed in parallel in a confocal detection scheme by a camera, allowing acquisition rates up to several hundred frames per second. Fast switching of the polarization state is provided by an electrooptical modulator placed in the excitation path. With this technique, fast confocal images can be recorded at any desired polarization angle, which offers the possibility to build polarimetric image stacks, in order to provide real-time monitoring of molecular order in dynamic specimens.

This article is organized as follows. Section II gives technical details on the setup, including the polarization control method. Section III describes the calibration protocol. Section IV details the data processing. Results obtained on two kinds of samples are reported in Sec. V. The acquisition rate as well as the use of multi-photon absorption are discussed in Sec. VI.

\section{DESCRIPTION OF THE SETUP}

\section{A. Optical setup}

The optical setup is presented in Fig. 1(d). It is based on a confocal spinning disk unit (CSU-X1-M1, Yokogawa) that is connected to the side-port of an inverted microscope (Eclipse Ti-U, Nikon). Laser excitation is provided by a continuouswave vertically-polarized 488-nm optically-pumped 
semiconductor laser (Sapphire 488-20, Coherent). Excitation power is adjusted by rotating a half-wave plate (WPH05M-488, Thorlabs) in front of a Glan polarizer (GL5-A, Thorlabs) having its transmission axis vertical. The laser beam is then sent into the polarization control unit (see Sec. II B), and then expanded using a $10 \times$ telescope (BE10, Thorlabs), in order to reach a transverse size that is comparable to the area of the pinhole array disk that is imaged onto the specimen. In order to minimize the number of optical elements on the excitation path, the collimation and shaping optics of the confocal spinning disk unit (CSU) were bypassed: a mirror in the excitation path of the CSU, which was accessible from outside, was removed, so that the laser beam could directly illuminate the microlens array disk of the CSU. The optical configuration of the CSU, which is detailed in Ref. 15, uses this microlens array disk in order to improve the illumination of the pinhole array disk, which is optically conjugated onto the sample plane, using an objective lens (Nikon Plan Apo VC $60 \times$, N.A. = 1.2 , water immersion) and an extra magnification of $1.5 \times$. This generates about 1000 excitation spots that illuminate simultaneously the specimen. The emitted fluorescence light is collected through the same objective lens, spatially filtered by the pinhole array disk, reflected by the dichroic mirror (Di01-T488-13×15×0.5, Semrock), spectrally filtered (bandpass 525/45) and imaged onto the camera (iXon 897 EMCCD, $512 \times 512$ pixels, Andor). Microlens and pinhole array disks rotate synchronously at a speed of $1800 \mathrm{rpm}$, and can generate a uniform scanning by a rotation of $30^{\circ}$ so that the exposure time of the camera needs to be an integral multiple of that time, namely $1 / 360=2.77 \mathrm{~ms}$. Note that there is no need to monitor or synchronize the rotation angle since any rotation of $30^{\circ}$, irrespectively to the start and stop position, provides an appropriate illumination. ${ }^{15}$

\section{B. Polarization control}

The polarization control is achieved by means of an electrooptical transverse modulator (EOM) (Pockels cell, model No 28-NP, Quantum Technology) followed by a quarter waveplate (WPQ05M-488, Thorlabs). Both elements are inserted such as their main axes make respective angles of $45^{\circ}$ and $0^{\circ}$ with respect to the input linear polarization. In this optical configuration, the output polarization state is linear, and is rotated by an angle, which is half of the phase retardation induced by the EOM. A rotation range of $180^{\circ}$ is, therefore, achievable by a retardation range of one wavelength, which corresponds to a typical high voltage range of $400 \mathrm{~V}$.

In order to ensure that any desired polarization state of the excitation could be properly delivered to the sample and not converted into elliptical states, the possible polarization distortions induced by the CSU have been investigated: a series of linear polarization states of various angles $\alpha$ has been generated using a polarizer, launched into the CSU, and the output light was analyzed by means of a home built polarimeter located in place of the objective lens. For each case, the polarimeter, which is described in the Appendix, provides the measured values the orientation $\Theta$ of the main axis of the el- (a)
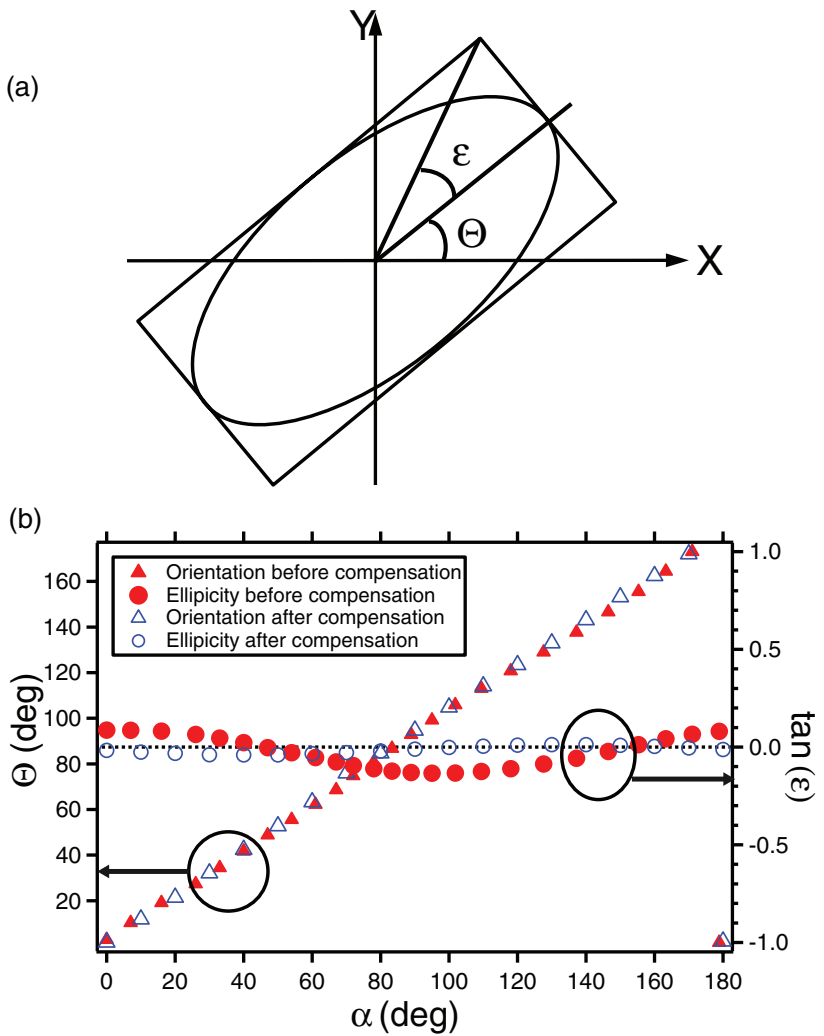

FIG. 2. (a) Definition of angles $\Theta$ and $\epsilon$ used to describe a general elliptical polarization state. (b) Polarimetric analysis of the excitation light after being transmitted through the CSU, as a function of the input linear polarization angle $\alpha$. Filled markers: before compensation. Empty markers: after compensation.

lipse and of its ellipticity $\tan (\epsilon)$, as defined by Fig. 2(a). The first results, which are plotted in Fig. 2(b) (filled markers), showed that the output light follows the same orientation than the input light, but the CSU induces a weak ellipticity at some angles. We found out that this response could be perfectly reproduced analytically by modeling the CSU (including transmission through the microlens array and the dichroic mirror, and reflections on the mirrors) as a single birefringent optical element having its fast axis oriented at $32.4^{\circ}$, a phase retardation of $12.3^{\circ}$, and no diattenuation. In order to compensate these distortions, a Soleil Babinet compensator (SBCVIS, Thorlabs) was finely oriented and tuned in retardation and introduced in the polarization control unit after the quarter waveplate (see Fig. 1(d)). By repeating the measurements after this compensation, it appears clearly (empty markers in Fig. 2(b)) that the appropriate polarization state is now delivered to the objective lens.

Similar investigations are needed in principle for the emission path. However, since fluorescence is only analyzed in terms of intensity, and not polarization, polarization distortions would not affect the measurement. The only necessary requirement is that the detection efficiency is not polarization dependent. A test was performed using white from a tungsten lamp was attenuated, collimated, linearly polarized, introduced in place of the objective lens, sent into the CSU (i.e., reflected on the dichroic mirror and other deflections mirrors), spectrally filtered, and measured with the camera. By rotating the input polarization, we have recorded output intensity 
variations of a few percents only, which were considered to be negligible.

\section{Data acquisition}

The data acquisition hardware is presented in Fig. 1(d). The command of the polarization control includes a highspeed analog output (AO) board (NI-USB-6351, National Instruments), which generates a control voltage in the $\pm 2.5 \mathrm{~V}$ range, followed by a waveform amplifier (WA301, Aim \& Thurlby Thander Instruments) that does not affect the voltage but increases the output power, and a high voltage amplifier (HVA-1M, Quantum Technology) which produces the high voltage $( \pm 200 \mathrm{~V})$ that is applied to the EOM, in addition to a bias of $200 \mathrm{~V}$. All these elements have a frequency response ranging from $\mathrm{DC}$ to $1 \mathrm{MHz}$. Moreover, the $\mathrm{AO}$ board uses direct memory access, so that the calculated sequence of command voltages is stored in a buffer, and then delivered without communication to the computer. This ensures fast switching between successive linear polarization states.

In order to ensure the shortest acquisition time together with an accurate synchronization between polarization control and the camera, the timing of the camera acquisition sequence has been carefully optimized: when the exposure time, an integral multiple of $1 / 360 \mathrm{~s}$, is set by the user to the camera, the minimum cycle time, which includes exposure time and delay to read the chip, is returned by the camera. This cycle time is set so that it is the duration of each polarization step, and therefore will determine the effective acquisition rate. In addition, the camera acquisition is externally trigged by a start pulse generated by the AO board at the starting of each sequence, and the image stack is stored in the camera buffer until it is transferred to the computer, after each stack acquisition.

Our camera can operate down to cycle times of $30 \mathrm{~ms}$, a value that is obtained for full definition operation and that can be reduced drastically using binning and/or using a cropped sensor mode. Ultimately, by using three polarization angles, up to about ten polarimetric full definition stacks can be recorded by the system every second.

\section{CALIBRATIONS}

\section{A. Polarization control voltage}

A full calibration curve that gives the relationship between voltage applied to the AO board and the polarization angle has been measured at the place of the objective lens, and is presented in Fig. 3. This reference curve allows to set accurately any desired polarization angle $\alpha$ to the system.

\section{B. Linearity of the camera response}

In order to ensure precise quantitative data analyses, the response of the camera has been carefully investigated. First, the analysis of the camera response in dark conditions shows a systematic offset of 380 as returned by the camera, irrespectively of the gain and exposure time. This offset will be

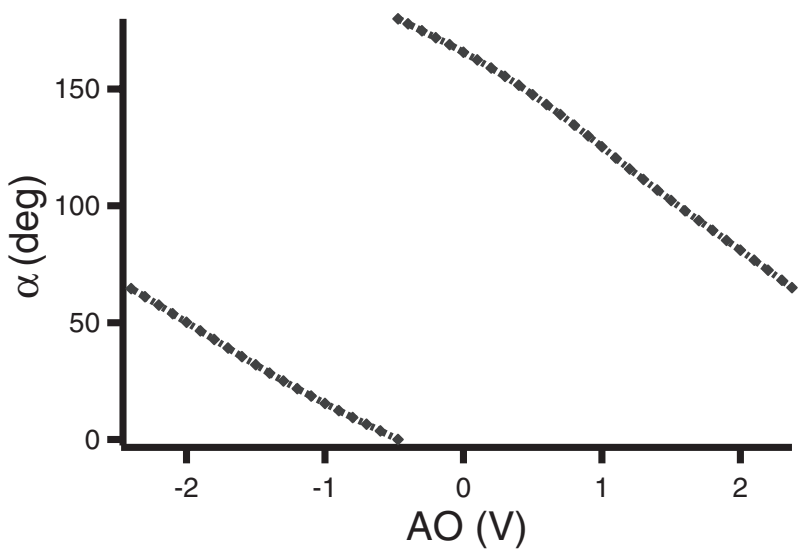

FIG. 3. Calibration of the excitation polarization angle vs. AO voltage.

systematically subtracted from all data acquisitions, including calibrations below.

In order to investigate the linearity of the camera response for given acquisition settings, fluorescence images of solutions of Rhodamine $6 \mathrm{G}$ in water, with concentrations ranging from a $1 \mu \mathrm{M}$ to $5 \mu \mathrm{M}$, have been recorded under various excitation power, ranging between $50 \mu \mathrm{W}$ and $7.5 \mathrm{~mW}$, in order to generate a wide range of signal. For every condition, a several regions of interest of $10 \times 10$ pixels have been selected, over which the response has been averaged. This analysis has been carried out for various gain settings, ranging between 100 and 1000. As it clearly appears in Fig. 4, we have observed a systematic threshold value of a measured signal (as encoded by the camera) of 6000 , above which the response of the camera versus excitation power is no longer linear. Note that since this threshold does not depend on the excitation power, it clearly indicates that the source of the saturation is not a saturation of fluorescence itself, but the camera readout process. Below this threshold, the linear relationship of recorded signal vs. the excitation power is verified, which guarantees that the measurements can be processed in a quantitative way without further signal correction.

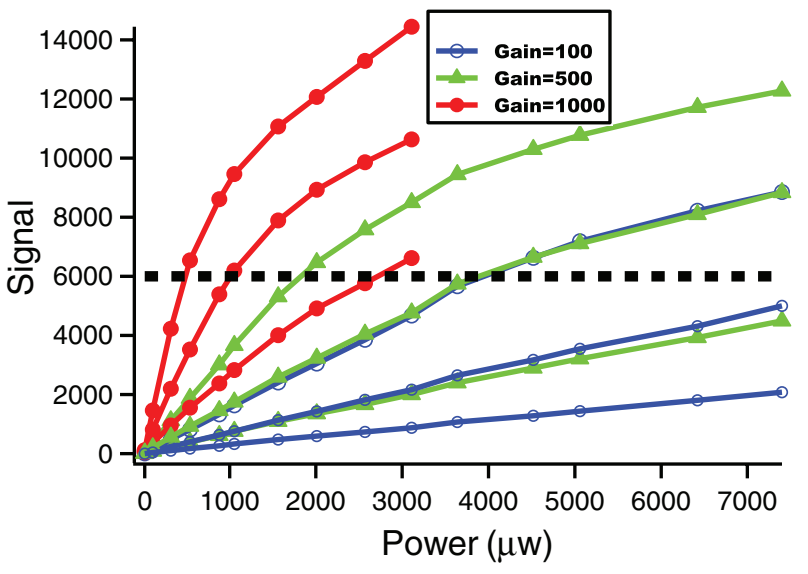

FIG. 4. Signal returned by the camera vs. excitation light power for different concentrations of solutions (connected data points) and different gain values. Response is linear below signal value of 6000 . 

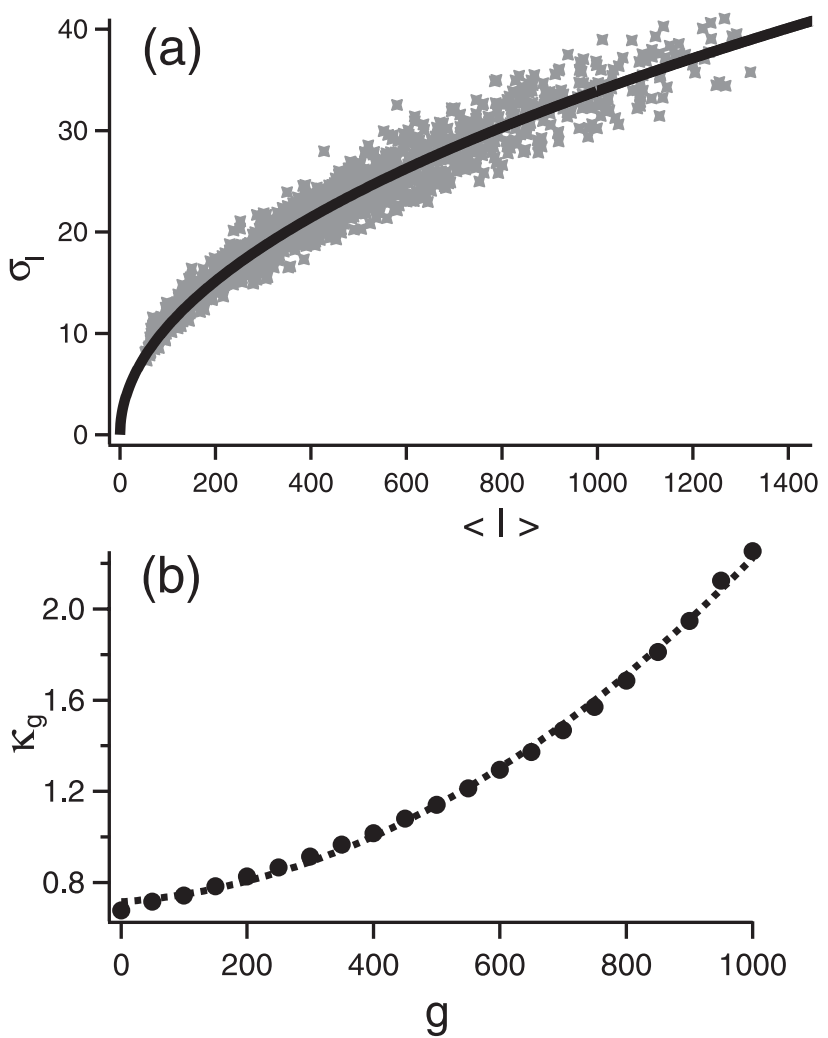

FIG. 5. Camera noise analysis. (a) Relationship between standard deviation $\sigma_{I}$ and mean value $\langle i\rangle$ for the case $g=600$. Square markers: data, black line: best fit using relationship $\sigma_{I}=\kappa_{g} \sqrt{\langle I\rangle}$, with here $\kappa_{g}=1.3$. (b) Dependence on the noise factor $\kappa_{g}$ as the function of gain $g$.

\section{Camera noise analysis}

The noise that affects the signal $I$ of the camera has been analyzed in details. Using, as above, a solution as fluorescent sample, various measurement conditions of exposure time and gain have been tested. For each case, 100 fluorescence images have been recorded, allowing to analyze systematically the standard deviation $\sigma_{I}$ as a function of the mean value $\langle I\rangle$ of the signal. A global analysis performed for many configurations shows that $\sigma_{I}$ depends on the mean signal according to $\sigma_{I}=\kappa_{g} \sqrt{\langle I\rangle}$, as illustrated in Fig. 5(a) for the case $g=600$. The factor $\kappa_{g}$ was found to be only dependent on the gain $g$, although $\langle I\rangle$ depends both on gain and exposure time. The dependence of $\kappa_{g}$ versus $g$ is plotted in Fig. 5(b). The consequences of the camera noise on the precision of the technique will be discussed in Sec. IV C.

\section{DATA PROCESSING}

\section{A. Theoretical model}

Using a similar formalism to what was developed in a framework of two photon absorption microscopy, ${ }^{16}$ the steady state fluorescence intensity emitted by an ensemble of dipoles is here written as

$$
\begin{aligned}
I_{\rho, \psi}(\alpha)= & \int_{0}^{2 \pi} \mathrm{d} \phi \int_{0}^{\pi} \mathrm{d} \theta \sin \theta|\vec{\mu}(\theta, \phi) \cdot \vec{E}(\alpha)|^{2} \\
& \times f_{\rho, \psi}(\theta, \phi) J(\theta, \phi) .
\end{aligned}
$$

This equation is a sum over all possible angles $(\theta, \phi)$ in space taken by an absorption transient dipole $\vec{\mu}$. The square modulus represents its absorption probability when excited by an electric field $\vec{E}$ having a linear state of polarization along an angle $\alpha$ with respect to the $X$ axis in the sample plane, as illustrated in the inset of Fig. 1(d). The function $J$ represents the detection probability of fluorescence and is needed in order to take into account the numerical aperture of the objective lens. $^{2}$

The modeling of the angular distributions of the dipoles uses a normalized distribution function $f_{\rho, \psi}$, that is chosen in accordance with the properties of the fluorescent probe and the geometry of the system to be imaged. In the present work, we address lipid membranes. For a lipid probe that would insert perpendicularly to the membrane, disorder induces a freedom of angles around this direction so that the orientational distribution can be modeled by a 3D filled cone of full aperture $\psi$, as illustrated in Fig. 1(b). Limiting the investigations to the equatorial plane of the cell/vesicle allows to assume that the cone axis lies in the sample plane, making an angle with respect to the $X$ axis that is denoted $\rho$. In the case of a fluorescent probe that lies on the membrane surface, it is more appropriate to use a pancake-like model, as illustrated in Fig. 1(c), with the corresponding definitions of $\rho$ and $\psi$.

For any set $(\rho, \psi), I_{\rho, \psi}$ has a sine dependence upon the angle $\alpha$ with a period of $180^{\circ}$, so it can be written as a truncated Fourier series ${ }^{13}$

$$
I_{\rho, \psi}(\alpha)=I_{0}[1+A(\rho, \psi) \cos (2 \alpha)+B(\rho, \psi) \sin (2 \alpha)],
$$

with

$$
\begin{gathered}
A(\rho, \psi)=\frac{2}{I_{0} \pi} \int_{0}^{\pi} \mathrm{d} \alpha I_{\rho, \psi}(\alpha) \cos (2 \alpha), \\
B(\rho, \psi)=\frac{2}{I_{0} \pi} \int_{0}^{\pi} \mathrm{d} \alpha I_{\rho, \psi}(\alpha) \sin (2 \alpha)
\end{gathered}
$$

and $I_{0}=\frac{1}{\pi} \int_{0}^{\pi} \mathrm{d} \alpha I_{\rho, \psi}(\alpha)$. Note here that $A(\rho, \psi)$ and $B(\rho$, $\psi)$ range between -1 and 1 . Moreover, one can show ${ }^{13}$ that any set $(\rho, \psi)$ corresponds to a unique set $[A(\rho, \psi), B(\rho, \psi)]$, as illustrated in Fig. 6 . This allows to compute numerically the inverse relationships $\rho(A, B)$ and $\psi(A, B)$.

As compare to other methods that are based on basic mathematical operations, ${ }^{11,12}$ this modeling approach may appear tedious. Nevertheless, this method is generic, so it can process any type and number of input polarization state or angular distribution. Moreover, we believe that the models (pancake or cone) that are used for decades to investigate lipid probe orientations ${ }^{2}$ provide a picture of the molecular order which is both comprehensive and quantitative.

\section{B. Data processing}

Images are recorded using a set of $N$ regularly-spaced polarization angles $\alpha_{k}$, where $k$ refers to the $k$ th image of the stack. Due to the sine shape of the response, three polarization with steps of $60^{\circ}$ are in principle enough for an 

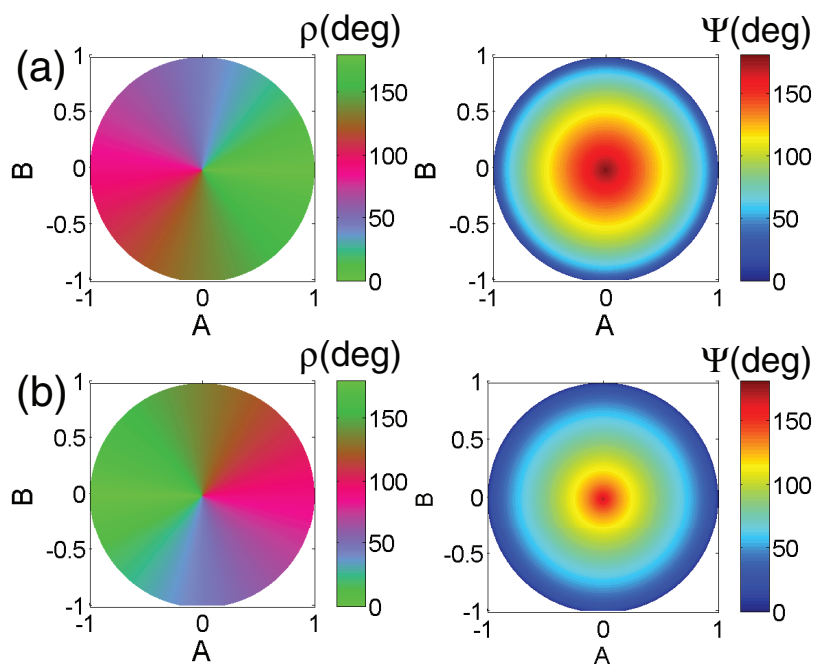

FIG. 6. Left: value of $\rho$ (colorscale) as a function of $(A, B)$. Dependence is purely azimutal. Right: value of $\psi$ (colorscale) as a function of $(A, B)$. Dependence is purely radial. (a) Cone model of Fig. 1(b), (b) pancake model of Fig. 1(c).

unambiguous retrieval of $\rho$ and $\psi$. However, a denser sampling, like a longer acquisition time, can improve the precision of the technique.

At each pixel of interest, the two coefficients $A$ and $B$ are computed according to

$$
\begin{aligned}
& A=\frac{2}{I_{0} N} \sum_{k=1}^{N} I\left(\alpha_{k}\right) \cos \left(2 \alpha_{k}\right), \\
& B=\frac{2}{I_{0} N} \sum_{k=1}^{N} I\left(\alpha_{k}\right) \sin \left(2 \alpha_{k}\right),
\end{aligned}
$$

where $I_{0}=\frac{1}{N} \sum_{k=1}^{N} I\left(\alpha_{k}\right)$. The values of the angles $\rho$ and $\psi$ are then retrieved using the inverse relationships $\rho(A, B)$ and $\psi(A, B)$, that has been built up numerically once for all values of $A$ and $B$ between -1 and 1 , with a sampling of 0.02 .

In practice, pixels of interest are selected by regions defined manually by the operator and on the basis on their total fluorescence $\sum_{k} I\left(\alpha_{k}\right)$. This allows to reject systematically image area where the low signal compromises the precision of the method. ${ }^{13}$

The validity of the retrieved set $(\rho, \psi)$ is assessed by calculating the normalized chi-square

$$
\chi^{2}=\frac{1}{N} \sum_{k=1}^{N} \frac{\left[I\left(\alpha_{k}\right)-I_{\text {theory }}\left(\alpha_{k}\right)\right]^{2}}{\sigma_{I}^{2}\left(\alpha_{k}\right)},
$$

where $I_{\text {theory }}\left(\alpha_{k}\right)$ is the reconstructed intensity dependence calculated by introducing the computed values of $A$ and $B$ in Eq. (2). In agreement with the camera noise analysis detailed in Sec. III C, the variance of every data point $\sigma_{I}^{2}$ is assumed to be $\kappa_{\mathrm{g}}^{2} I\left(\alpha_{k}\right)$. According to our experience, data with $\chi^{2}<1.5$ always showed a good agreement between measurement and theory. In practice, values of $\chi^{2}>1.5$ are due to instabilities such as sample drift, membrane local motion, photobleaching, etc., and are systematically rejected.

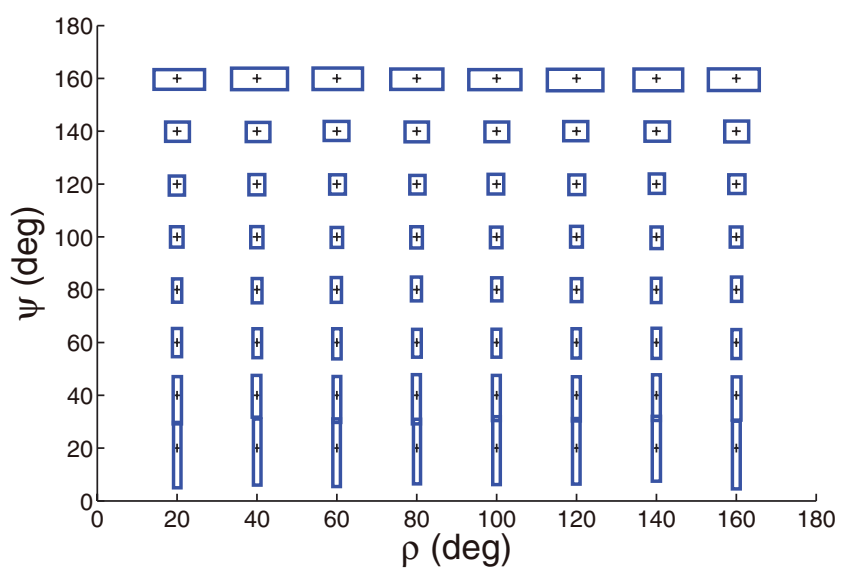

FIG. 7. Precision analysis. For each set $(\rho, \psi)$ (indicated by a cross, the rectangle represents error bars on $\rho$ (rectangle width is twice the standard deviation on $\rho$ ) and $\psi$ (rectangle height is twice the standard deviation on $\psi)$. See text for simulation parameters.

The whole data processing takes about $100 \mathrm{~ms}$ on a standard personal computer.

\section{Precision analysis}

The precision of the method has been investigated using Monte Carlo simulations. For each set $(\rho, \psi)$, the theoretical response $I\left(\alpha_{k}\right)$ has been computed for the cone model (Fig. 1(b)) using Eq. (1), for six angles $\alpha_{k}$ from $\alpha_{1}=0^{\circ}$ to $\alpha_{6}=150^{\circ}$ and scaled in order to simulate a total fluorescence signal $I_{\text {tot }}=2000$, using a camera gain of $g=500$. These values correspond to typical acquisition parameters. Using a normally-distributed random-number generator, 500 artificial datasets have been simulated and processed using the method described above. The distribution of the retrieved values of $\rho$ and $\psi$ values have been statistically analyzed.

The result are summarized in Fig. 7, where each nominal set $(\rho, \psi)$ is represented as a cross. The rectangle quantifies the distribution of the retrieved values $\rho$ and $\psi$. The center of the rectangle is the mean value, and its width (and height) is twice the standard deviation on $\rho$ (and $\psi$, respectively). These results show an uncertainty on $\psi$ of about $5^{\circ}$. This precision is slightly better for large $\psi$ values, but does not depend on the actual $\rho$. The uncertainty on $\rho$ is in the range of the degree, except for large values of $\psi$ for which orientation becomes meaningless. Simulations carried out for various $I_{\text {tot }}$ (not presented here) show that the obtained uncertainty on both $\rho$ and $\psi$ are simply scaled according to $\sqrt{I_{\text {tot }}}$, irrespectively on the number of angle steps. Therefore, increasing exposure time, using smaller angle steps, or binning neighbor pixels, are appropriate ways to improve the precision. Note that, although not presented here, comparable results have been obtained using the pancake model of Fig. 1(c).

Note that camera gain provides another powerful way to increase the signal. However, the effect of gain on signal and noise is specific to the camera used, so a systematic study on a given device is out of the scope of the present article. For the sake of clarity and generality, these simulations were restricted to a unique value of camera gain. 


\section{RESULTS}

\section{A. Giant unilamellar vesicles}

The technique has been tested preliminarily on giant unilamellar vesicles (GUVs), which constitute a welldocumented model systems. ${ }^{17-20}$ GUVs made of 1,2-dioleoyl$s n$-glycero-3-phosphocholine (DOPC) and dye (concentration 1\%) mixtures, with diameters ranging from 10 to $80 \mu \mathrm{m}$, were prepared by electroformation ${ }^{21}$ using the method detailed in Ref. 16. The dye that we used was DiIC ${ }_{18}(3)$ (Invitrogen), a lipophilic membrane stain that lies on the membrane surface, ${ }^{2}$ and whose angular distribution can be described using the pancake model of Fig. 1(c).

The sample was probed by 30 polarization angles from $\alpha_{1}=0^{\circ}$ to $\alpha_{30}=174^{\circ}$. Camera gain was set to $g=1000$, exposure time was set to $33.3 \mathrm{~ms}$, cycle time was $72 \mathrm{~ms}$, so the whole image stack was acquired in about $2.5 \mathrm{~s}$. The total fluorescence image obtained on the equatorial plane of a GUV of diameter $15 \mu \mathrm{m}$ is shown in Fig. 8(a). As expected from a confocal system, the center of GUV appears dark, a signature of good sectioning capabilities. Figure 8(b) shows the data corresponding to point labeled as A in Fig. 8(a), as well as the theoretical response corresponding to the retrieved parameters $\rho$ and $\psi$. The post processing of a whole image stack takes about $100 \mathrm{~ms}$, and the obtained result is shown in Fig. 8(c) (cf. Video 1), which is a composite image built by superimposing a gray-scale image of total fluorescence and colored sticks for every processed pixel. Each stick is oriented with the retrieved angle $\rho$, while its color gives the value of $\psi$. Such picture is displayed in real time to the user, with a frame rate of $0.4 \mathrm{fps}$.

These results show clearly that the angle $\rho$ is perpendicular to the membrane, i.e., the fluorescent dipole lies on the membrane. As expected for this system, made of a unique molecular species, the value of the angle $\psi$ is homogeneous over the membrane contour, showing remarkably that the technique addresses with the same efficiency all membrane orientations. The average value of $\psi$ of $72^{\circ}$ is in agreement with previous measurements. ${ }^{13}$ The histogram in Fig. 8(c) (cf. Video 1) shows the narrow distribution of the values.

Note that in the present case, the main source of the obtained standard deviation of $9^{\circ}$ is the uncertainty of the method. Indeed, numerical simulations have been run, in order to generate an artificial dataset of an ideal GUV having a single value $\psi=72^{\circ}$, assuming the same measurement conditions (angle steps, total intensity, camera gain, etc.). The result after processing is reported in Fig. 8(d), with a standard deviation comparable to what was observed on a real sample.

\section{B. COS-7 cells}

Compared to GUVs, the morphology of cell membranes is usually more complex, due to sub-resolution morphological features, ${ }^{13}$ so that polarization-resolved microscopy can show its power. We use here COS-7 cells that have been labeled by di-8-ANEPPQ, a membrane potential dye that inserts perpendicularly to the membrane, ${ }^{16}$ so that possible orientations can be described by our cone model. The samples were prepared
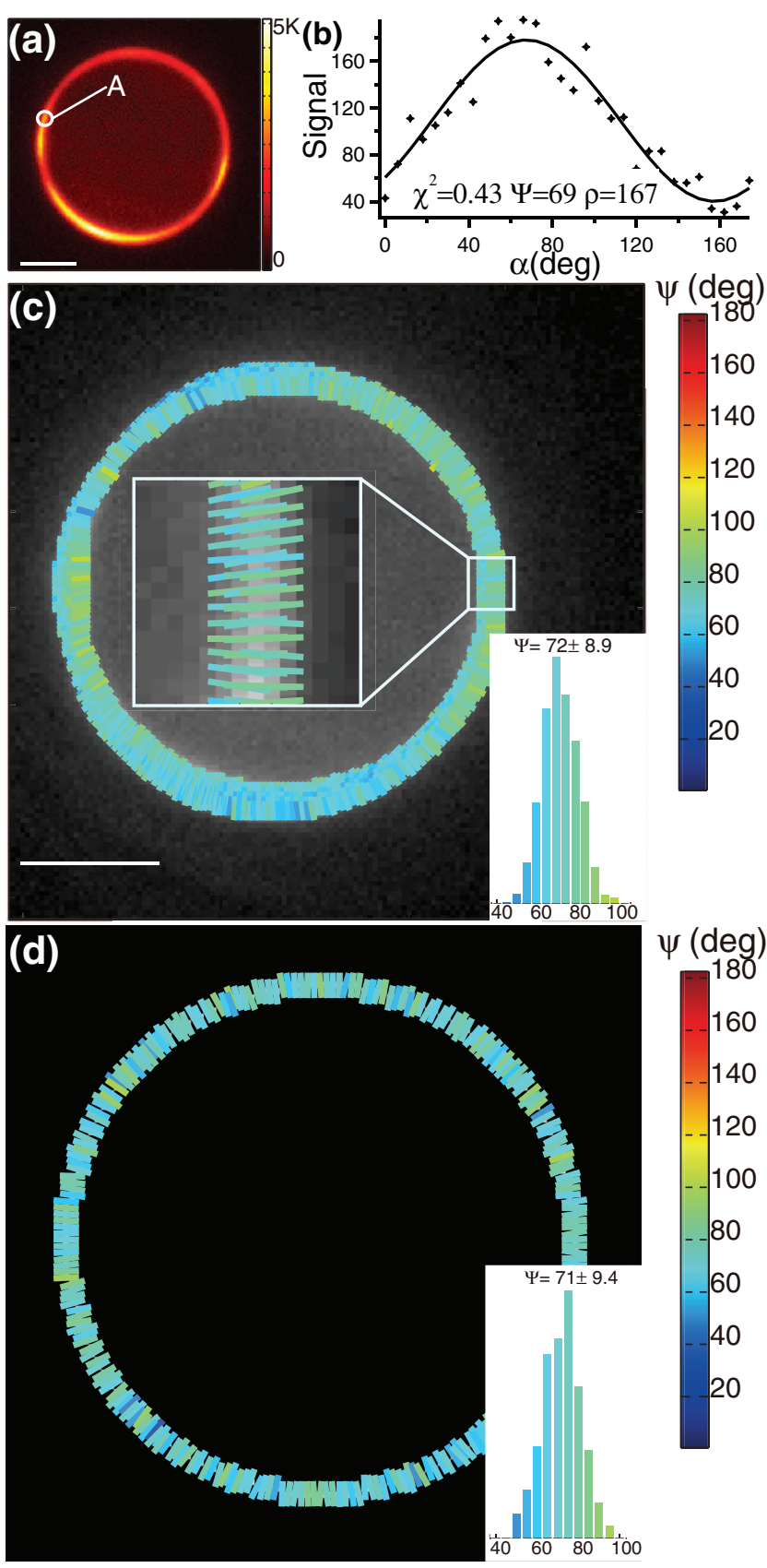

FIG. 8. Results of measurements performed on a GUV labeled with DiIC 18 . (a) Image of the total fluorescence intensity $I_{\text {tot }}$ (b) An example of dataset extracted at the point indicated by a circle in Fig. 8(a). (c) Experimental composite image showing the mean orientation $\rho$ (indicated by an orientated stick) and the angular aperture $\psi$ (ranging from $0^{\circ}$ to $180^{\circ}$ and encoded as a color) of the pancake model used to describe the orientation of the fluorophore. The histogram shows the distribution of $\psi$ values. Scale bar is $5 \mu \mathrm{m}$. (d) Numerical simulation of data processing for an artificial dataset mimicking a GUV. Parameters are $\psi=72^{\circ}, I_{\text {tot }}=2000, g=1000$ (enhanced online). [URL: http://dx.doi.org/10.1063/1.4807318.1]

following the protocol detailed in Ref. 16. Imaging was performed at room temperature.

Measurements have been carried on cells with the same acquisition parameters as for GUVs of Sec. V A. The focus was set in an intermediate plane in order to ensure that the fluorescent molecules lie in average in the sample plane. An example of measurement for a typical cell is shown in Fig. 9. Here again, dipoles are clearly perpendicular to the 


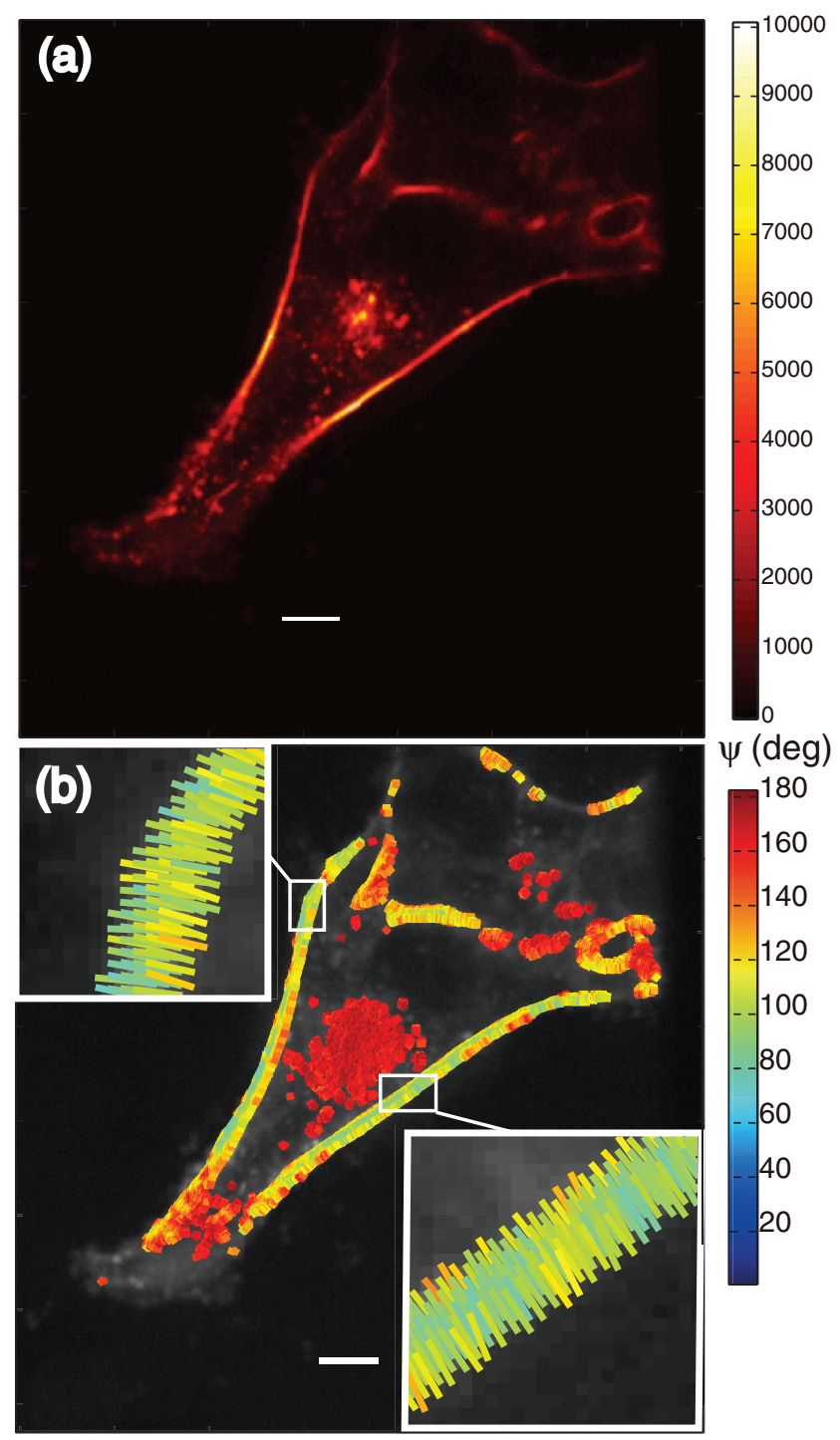

FIG. 9. Experimental results on COS-7 cells labeled with di-8-ANEPPQ. (a) Fluorescence image. The colorscale indicates the total intensity $\sum_{k} I\left(\alpha_{k}\right)$. (b) Composite image summarizes the mean orientation $\rho$ (indicated by an orientated stick) and the angular aperture $\psi$ (ranging from $0^{\circ}$ to $180^{\circ}$, encoded as a color). Insets are close-ups. Scale bars are $5 \mu \mathrm{m}$.

membrane. Similar to what was observed in previous work, the values of $\psi$ recorded on the membrane allow to separate clearly regions of high order $\left(\psi \approx 80^{\circ}\right)$ and low order $\left(\psi \approx 150^{\circ}\right)$ along the contour which can be explained by possible sub-resolution morphological features. ${ }^{13}$ A more homogeneous response is obtained in the less ordered cytoplasm regions, where $\psi \approx 180^{\circ}$ indicates an isotropic angular distribution of fluorophores.

\section{DISCUSSION AND CONCLUSION}

These examples show that our technique allows to retrieve the values of $\rho$ and $\psi$ at a rate of $0.4 \mathrm{fps}$, a value which is significantly lower than the acquisition rate of $10 \mathrm{fps}$ claimed in Sec. II C. This is mainly due to the high precision that was desired for $\rho$ and $\psi$, together with the relatively low brightness of the fluorophores that we have used in this work.
More generally, for a given setup and sample, strategies to improve the acquisition rate include: (i) reducing the number of polarization angles or the exposure time, which will also reduce the precision of the retrieved parameters, as discussed in Sec. IV C, (ii) reducing the number of pixel to be measured, by means of pixel binning or sensor cropping, which does not affect the precision. In practice, it appears that the acquisition rate is much more limited by the fluorophore brightness and camera signal to noise ratio than by the polarization switching or CSU velocity.

Although the instrument presented here was based on one-photon absorption, a similar development could be carried out using multi-photon excitation. This latter could be indeed a relevant choice in the case of weakly oriented distributions (i.e., $\psi \approx 60^{\circ}-140^{\circ}$ ), for which the more directed photo-selectivity of multiphoton excitation would provide a better precision ${ }^{4}$ on the retrieved parameters. However, the choice of multi-photon vs. one-photon excitation should not be motivated by the need to investigate thick samples, as it is often the case for imaging. Indeed, the multipoint excitation strategy of the CSU makes it intrinsically sensitive to pinhole cross-talk and, therefore, limited to thin samples. The case of multipoint excitation of thick scattering samples deserves a specific instrumental development, either using a CSU with increased interpinhole distances, ${ }^{22}$ or a beam multiplexing system. ${ }^{4,23}$

In conclusion, this technique clearly improves the acquisition rate of angle-resolved LD data, by making possible high precision measurements at the timescale of the second, opening the door to more dynamic samples. Together with the highly parallel acquisition, the spinning disk confocal geometry offers the benefit of an axial resolution which is similar to what is obtained using conventional scanning confocal microscopy, ${ }^{15}$ and therefore can address complex 3D biomolecular objects.

\section{ACKNOWLEDGMENTS}

This research was funded by the Région Provence Alpes Côte d'Azur and by the French Agence Nationale de la Recherche under Contracts ANR-2010-BLAN-150902 and ANR-10-INBS-04-01 (France-BioImaging Infrastructure network). X. W. received a scholarship from the China Scholarship Council. The authors are grateful to Julien Duboisset and Hervé Rigneault for stimulating discussions and to Julien Savatier for his assistance in sample preparation.

\section{APPENDIX: MEASUREMENT OF POLARIZATION STATE}

Polarization states of the system have been analyzed using the method of the rotating quarter wave plate. ${ }^{24}$ The light to be analyzed is sent onto a quarter wave plate, whose fast axis makes an angle $\Omega$ with respect to direction $X$, followed by a vertical polarizer and a photodetector.

It is easy to show, for instance, using the Jones formalism, that the intensity $I(\Omega)$ measured by the detector can be written 
as

$$
\begin{aligned}
I(\Omega)= & I_{0}\left[\frac{1}{2}-\frac{1}{4} \cos 2 \epsilon \cos 2 \Theta-\frac{1}{2} \sin 2 \epsilon \sin 2 \Omega\right. \\
& -\frac{1}{4} \cos 2 \epsilon \cos 2 \Theta \cos 4 \Omega \\
& \left.-\frac{1}{4} \cos 2 \epsilon \sin 2 \Theta \sin 4 \Omega\right],
\end{aligned}
$$

where $\Theta$ is the main axis of the ellipse and $\tan \epsilon$ is the ellipticity of the incident light, which is described here as a general elliptical polarization state, as shown in Fig. 2(a). Equation (A1) can be written as a truncated Fourier series

$$
I(\Omega)=\sum_{k=0}^{+\infty}\left[p_{k} \cos k \Omega+q_{k} \sin k \Omega\right],
$$

the only non-zero coefficients being

$$
\begin{aligned}
& p_{0}=\frac{I_{0}}{2}-\frac{I_{0}}{4} \cos 2 \epsilon \cos 2 \Theta=\frac{I_{0}}{2}+q_{4}, \\
& q_{2}=-\frac{I_{0}}{2} \sin 2 \epsilon \\
& p_{4}=-\frac{I_{0}}{4} \cos 2 \epsilon \cos 2 \Theta \\
& q_{4}=-\frac{I_{0}}{4} \cos 2 \epsilon \sin 2 \Theta .
\end{aligned}
$$

These coefficients can be easily extracted from the experimental values $I(\Omega)$ using basic Fourier algebra

$$
\begin{aligned}
& p_{k}=\frac{2}{N} \sum_{p=1}^{N} I\left(\Omega_{p}\right) \cos k \Omega_{p}, \\
& q_{k}=\frac{2}{N} \sum_{p=1}^{N} I\left(\Omega_{p}\right) \sin k \Omega_{p},
\end{aligned}
$$

for $k=2,4$ and $p_{0}=\frac{1}{N} \sum_{p=1}^{N} I\left(\Omega_{p}\right)$, where $N$ is the number of discrete regularly spaced measured angles $\Omega_{p}$. Then, one can show that

$$
\Theta=\frac{1}{2} \arctan \left(\frac{q_{4}}{p_{4}}\right)+n \pi / 2,
$$

where $n$ is an integer. Because $-\frac{\pi}{2} \leq \epsilon \leq \frac{\pi}{2}, \cos 2 \epsilon \geq 0, n$ is chosen so that both $\cos 2 \Theta$ with $p_{4}$, and $\sin 2 \Theta$ with $p_{4}$ are opposite in sign. Finally, $\epsilon$ is given by

$$
\epsilon=\frac{1}{2} \arctan \left(\frac{q_{2} \sin 2 \Theta}{2 q_{4}}\right) .
$$

${ }^{1}$ G. Weber, Adv. Protein Chem. 8, 415 (1953).

${ }^{2}$ D. Axelrod, Biophys. J. 26, 557 (1979).

${ }^{3}$ A. M. Vrabioiu and T. J. Mitchison, Nature (London) 443, 466 (2006).

${ }^{4}$ R. K. P. Benninger, B. Önfeld, M. A. A. Neil, D. M. Davies, and P. M. W. French, Biophys. J. 88, 609 (2005).

${ }^{5}$ G. Steinbach, I. Pomozi, O. Zsiros, L. Menczel, and L. Garab, Acta Histochem. 111, 317 (2009).

${ }^{6}$ J. E. Reeve, A. D. Corbett, I. Boczarow, T. Wilson, H. Bayley, and H. L. Anderson, Biophys. J. 103, 907 (2012).

${ }^{7}$ J. Lazar, A. Bondar, S. Timr, and S. J. Firestein, Nat. Methods 8, 684 (2011).

${ }^{8}$ J. Borejdo and S. Burlacu, Biophys. J. 65, 300 (1993).

${ }^{9}$ K. Florine-Casteel, Biophys. J. 57, 1199 (1990).

${ }^{10}$ J. V. Rocheleau, M. Edidin, and D. W. Piston, Biophys. J. 84, 4078 (2003).

${ }^{11}$ B. S. DeMay, N. Noda, A. S. Gladfelter, and R. Oldenbourg, Biophys. J. 101, 985 (2011)

${ }^{12}$ J. F. Lesoine, J. Y. Lee, J. R. Krogmeier, H. Kang, M. L. Clarke, R. Chang, D. L. Sackett, R. Nossal, and J. Hwang, Rev. Sci. Instrum. 83, 053705 (2012).

${ }^{13}$ A. Kress, X. Wang, H. Ranchon, J. Savatier, H. Rigneault, P. Ferrand, and S. Brasselet, "Mapping the local organization of cell membranes using excitation polarization resolved confocal fluorescence microscopy," Biophys. J. (submitted).

${ }^{14}$ J. Duboisset, P. Ferrand, W. He, X. Wang, H. Rigneault, and S. Brasselet, J. Phys. Chem. B 117, 784 (2013).

${ }^{15}$ H. Hirukawa, H. Nakayama, Y. Tanibata, and Y. Kuwabara, Yokogawa Technical Report No. 45/2008, English edition, p. 21.

${ }^{16}$ A. Gasecka, T.-J. Han, C. Favard, B. R. Cho, and S. Brasselet, Biophys. J. 97, 2854 (2009).

${ }^{17}$ C. Dietrich, L. A. Bagatolli, Z. N. Volovyk, N. L. Thompson, M. Levi, K. Jacobson, and E. Gratton, Biophys. J. 80, 1417 (2001).

${ }^{18}$ A. V. Samsonov, I. Mihalyov, and F. S. Cohen, Biophys. J. 81, 1486 (2001).

${ }^{19}$ D. Scherfeld, N. Kahya, and P. Schwille, Biophys. J. 85, 3758 (2003).

${ }^{20}$ T. Baumgart, S. T. Hess, and W. W. Webb, Nature (London) 425, 821 (2003).

${ }^{21}$ M. I. Angelova and D. S. Dimitrov, Faraday Discuss. Chem. Soc. 81, 303 (1986).

${ }^{22}$ T. Shimozawaa, K. Yamagatab, T. Kondoc, S. Hayashic, A. Shitamukaid, D. Konnod, F. Matsuzakid, J. Takayamae, S. Onamie, H. Nakayamaf, Y. Kosugif, T. M. Watanabeg, K. Fujitai, and Y. Mimori-Kiyosuea, Proc. Natl. Acad. Sci. U.S.A. 110, 3399 (2013).

${ }^{23}$ J. Bewersdorf, R. Pick, and S. W. Hell, Opt. Lett. 23, 655 (1998).

${ }^{24}$ E. Collett, Polarized Light. Fundamentals and Applications (Marcel Dekker, New York, 1993). 